\title{
643.
}

\section{ON A QUARTIC CURVE WITH TWO ODD BRANCHES.}

[From the Messenger of Mathematics, vol. vi. (1877), pp. 107, 108.]

IT is a known theorem that the branches of a plane curve are even or odd; viz. two even branches, or an even and an odd branch (whether of the same curve or of different curves) intersect in an even number (it may be 0 , and this is to be understood throughout) of real points; but two odd branches (of the same curve or of different curves) intersect in an odd number of real points*.

In particular, a right line is an odd branch, and hence it meets any even branch of a curve in an even number of real points, and an odd branch in an odd number of real points; or (what is the same thing) an even branch is one which is met by any right line whatever in an even number of real points; and an odd branch is one that is met by any right line whatever in an odd number of real points.

It is to be observed, that the simple term branch is used to denote what has been called a complete branch, viz. the partial branches which touch an asymptote at its opposite extremities are considered as parts of one and the same branch, and so in other cases. Thus a quadric curve, whether ellipse, parabola, or hyperbola, is one even branch; a cubic curve is either one odd branch, or else it is an odd branch and an even branch; and generally a curve of an odd order has always an odd number of odd branches, and a curve of an even order has always an even number of odd branches.

A curve without nodes has at most one odd branch; for if there were two, these would intersect in a real point, which would be a real node on the curve. In particular, a quartic curve having two odd branches must have a real node; this however may be, as in the instance about to be given, a node at infinity.

A simple instance of a quartic curve with two odd branches is that represented by the equation

$$
\left(x^{2}-1\right)\left(y^{2}+1\right)-2 m x y=0,
$$

* The two branches must be distinct branches; a branch whether odd or even does not of necessity intersect itself (have upon it any real node), but it may intersect itself in an odd, or an even, number of real points. 
or, what is the same thing,

where

$$
y=\frac{1}{x^{2}-1}\left[m x \pm \sqrt{ }\left\{-\left(x^{2}-\alpha^{2}\right)\left(x^{2}-\frac{1}{\alpha^{2}}\right)\right\}\right]
$$

or say

$$
\alpha^{2}+\frac{1}{\alpha^{2}}=2+m^{2}
$$

$$
\begin{aligned}
& \alpha^{2}=\frac{1}{2}\left\{2+m^{2}+m \sqrt{ }\left(4+m^{2}\right)\right\}, \\
& \frac{1}{\alpha^{2}}=\frac{1}{2}\left\{2+m^{2}-m \sqrt{ }\left(4+m^{2}\right)\right\},
\end{aligned}
$$

so that $m$ being positive $\alpha>1$, and the curve consists of two real branches included between the lines $x=\alpha, x=\frac{1}{\alpha}$, and the lines $x=-\alpha, x=-\frac{1}{\alpha}$ respectively; each of these lines touches the curve in a real point, viz. $x$ having any one of the last-mentioned values, the value of $y$ at the point of contact is $y=\frac{m x}{x^{2}-1}$; and between each pair of lines we have the asymptote $x=+1$ or $x=-1$. Hence the curve has the form shown in the figure, and it is thereby evident, that each branch of the curve is met by

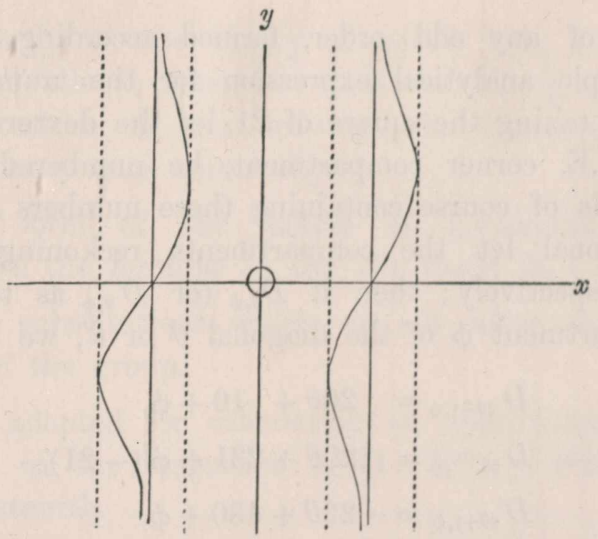

any real right line whatever in one real point, or else in three real points. The numerical values in the figure are $\alpha=\frac{3}{2}, m=\frac{5}{6}$, whence also $x=\alpha$ or $-\frac{1}{\alpha}, y=1$, and $x=-\alpha$ or $\frac{1}{\alpha}, y=-1$.

The curve has two nodes at infinity, viz. writing the equation in the form

that is,

$$
\left(x^{2}-z^{2}\right)\left(y^{2}+z^{2}\right)-m x y z^{2}=0,
$$

$$
x^{2} y^{2}+z^{2}\left(x^{2}-y^{2}-m x y\right)+z^{4}=0,
$$

it appears that the points $(z=0, x=0),(z=0, y=0)$ are each of them a node. The first of these $(z=0, x=0)$ is the real intersection of the two odd branches: the other of them is a conjugate point. 